\title{
Avaliação do nível mínimo de audição em lactentes de seis a 24 meses por meio do reforço visual
}

\author{
Measurement of the minimal hearing level in infants from six to \\ 24 months through visual reinforcement
}

\author{
Isabel Cristina Cavalcanti Lemos ${ }^{1}$, Tatiana Tomé ${ }^{2}$, Jéssika Nunes Gomes da Silva ${ }^{3}$, José Roberto Pereira Lauris ${ }^{4}$, \\ Andréa Cintra Lopes ${ }^{5}$
}

\begin{abstract}
RESUMO
Objetivo: Investigar, em campo livre, o comportamento auditivo de lactentes ouvintes de seis a 24 meses de idade cronológica, quanto aos aspectos de duração da avaliação, nível mínimo de resposta e análise crítica do método utilizado. Métodos: Foram avaliados 30 lactentes, de ambos os gêneros, subdivididos em grupos etários: Grupo I - 6 meses a 11 meses 29 dias (n=11); Grupo II - 12 meses a 17 meses 29 dias ( $n=9)$; Grupo III - 18 meses a 24 meses ( $n=10)$. Foram avaliadas as frequiências de 500, 1000, 2000 e 4000 Hz, bilateralmente, não sequienciais, em campo livre. Resultados: A mediana do nível mínimo de audição foi de 30 dB em todas as frequiências e todos os grupos. Observou-se que os Grupos I e III tiveram maior variabilidade dos níveis mínimos de audição enquanto o Grupo II mostrou-se mais homogêneo. O Grupo I necessitou de maior tempo de condicionamento, apresentando cansaço, agitação e tempo de atenção reduzido sendo necessário maior número de interrupções no exame. Já o Grupo III evidenciou menor motivação para responder ao teste, já que a técnica do reforço visual não é tão interessante nessa faixa etária, fazendo com que o desinteresse pelo reforço aconteça mais rapidamente. Estes aspectos não inviabilizam o procedimento, no entanto requerem maior experiência dos avaliadores. Conclusões: O método mostrou-se eficiente para a faixa etária pesquisada, não somente como um método de triagem, mas também para auxiliar a investigação do nível mínimo de audição, em campo livre, durante o processo diagnóstico.
\end{abstract}

Descritores: Percepção auditiva/métodos; Comportamento do lactente; Técnicas de diagnóstico otológicos; Estimulação visual; Lactente

\section{INTRODUÇÃO}

Muitos comitês internacionais e nacionais têm indicado

(1) Acadêmica do Curso de Mestrado em Fonoaudiologia do Departamento de Fonoaudiologia da Faculdade de Odontologia de Bauru da Universidade de São Paulo - USP - Bauru (SP), Brasil.

(2) Especializanda em Audiologia Clínica e Educacional do Hospital de Reabilitação de Anomalias Craniofaciais da Universidade de São Paulo USP - Bauru (SP), Brasil.

(3) Fonoaudióloga pela Faculdade de Odontologia de Bauru da Universidade de São Paulo - FOB-USP - Bauru (SP), Brasil.

(4) Professor Doutor do Departamento de Saúde Coletiva da Faculdade de Odontologia de Bauru da Universidade de São Paulo - USP - Bauru (SP), Brasil.

(5) Professora Doutora do Departamento de Fonoaudiologia da Faculdade de Odontologia de Bauru da Universidade de São Paulo - USP - Bauru (SP), Brasil.

Trabalho desenvolvido no Departamento de Fonoaudiologia da Faculdade de Odontologia de Bauru da Universidade de São Paulo - USP - Bauru (SP), Brasil, com apoio financeiro da FAPESP.

Endereço para correspondência: Andréa Cintra Lopes. Departamento de Fonoaudiologia - Al. Octavio Pinheiro Brisolla, 9-75, Bauru - SP, CEP 17012901. E-mail: aclopes@fob.usp.br

Recebido em: 18/4/2006; Aceito em: 15/5/2007 os programas de triagem auditiva neonatal e são unânimes em recomendar que toda criança deve ser avaliada auditivamente até os três meses de idade, por meio de técnicas objetivas, e por meio de audiometrias comportamentais a partir de cinco meses de idade, propiciando que possíveis alterações sejam detectadas e que ocorra a intervenção.

Há uma crescente preocupação dos profissionais envolvidos com crianças, em identificar o mais cedo possível as alterações auditivas ${ }^{(1-3)}$, o que favorece a eficiência dos tratamentos de (re)habilitação. Neste contexto, a avaliação audiológica de crianças pequenas, nos últimos anos atingiu significativos progressos no que diz respeito a métodos, técnicas e tecnologia ${ }^{(4-8)}$. Esses avanços ocorreram tanto no que se refere aos métodos eletrofisiológicos quanto aos comportamentais de avaliação da audição.

A avaliação auditiva subjetiva convencional (audiometria) só pode ser realizada em crianças a partir de dois a três anos de idade, com o método do condicionamento. Aos dois anos de idade, cerca de $50 \%$ das crianças estão aptas a estabelecer níveis mínimos em três frequiências e, a partir de três anos, $75 \%$ estabelecem limiares em seis frequiências ou mais ${ }^{(9)}$. 
O condicionamento consiste na realização de um comportamento voluntário após a apresentação de um dado estímulo, devendo esse comportamento ser reforçado positivamente para que se mantenha e/ou se repita ${ }^{(10-11)}$. Assim, a audiometria lúdica condicionada é aquela em que a criança é solicitada a realizar uma atividade lúdica ante um estimulo sonoro $^{(12-13)}$. Dentre os procedimentos subjetivos para avaliar a audição de crianças até três anos de idade temos as técnicas com e sem o condicionamento. Na Audiometria do Reflexo de Orientação Condicionada (CORA) se observa a busca do estimulo pela criança e só se aceita como resposta a localização da fonte sonora (reflexo de orientação), nesta técnica o reforço visual é um brinquedo iluminado e a faixa etária é de 12 a 36 meses de idade ${ }^{(14)}$. Dando seguimento a esta técnica, uma nova técnica foi elaborada e denominada Audiometria de Reforço Visual (VRA), baseada na CORA, onde se consideram como respostas aos estímulos o comportamento reflexo, a resposta de investigação, a resposta de orientação ou as respostas espontâneas. Aqui os reforços visuais utilizados são brinquedos iluminados ou slides de figuras projetadas e a faixa etária é de seis meses a três anos de idade $^{(15)}$. Outra técnica, sem a utilização do condicionamento, é a Audiometria de Observação Comportamental (BOA), que consiste em observar o comportamento da criança diante da apresentação do estimulo sonoro. Este procedimento é utilizado em neonatos e lactentes até seis meses de idade ${ }^{(16)}$. Ainda temos a Audiometria de Reforço Visual Informatizada (IVRA), que é a mesma técnica da VRA, mas utiliza um equipamento controlado por um sistema informatizado, o que promove maior objetividade ao teste. Ele pode ser aplicado na faixa etária de seis a 24 meses e o reforço visual são animais de pelúcia que se iluminam e movimentam-se ${ }^{(17)}$.

O PA5 marca Interacoustics, é um audiômetro pediátrico, portátil, em campo livre, de fácil manuseio e com reforço visual para avaliação clínica do comportamento auditivo de crianças nos aspectos de duração da avaliação e nível mínimo de resposta de cada faixa etária.

No Brasil, tudo indica que o primeiro padrão de desenvolvimento da função auditiva foi proposto em 1995. Neste estudo, os autores investigaram 499 crianças de zero a 13 meses, atendidas na disciplina de Pediatria Neonatal, Puericultura e Pediatria Social e Distúrbios da Audição da UNIFESP, no período de 1988 a 1992 e apresentaram a referencia do desenvolvimento da função auditiva em população brasileira. Em sua revisão de literatura foi possível obter os valores médios dos níveis mínimos de respostas obtidos em crianças saudáveis. Estes se encontram entre 60 e 80 dBNA nas crianças de zero a quatro meses, entre 40 e 50 dBNA nas crianças de quatro a seis meses, entre 30 e $40 \mathrm{~dB}$ nas de seis a nove meses e entre 20 e 40 dBNA nas de nove a 12 meses $^{(18)}$. As etapas de desenvolvimento da audição foram divididas em dois grupos de crianças durante o primeiro ano de vida: crianças nascidas a termo sem intercorrências e crianças nascidas pré-termo. Este último grupo ainda foi subdividido em crianças com seqüela neurológica e sem seqüela neurológica. Para tanto, foram utilizadas diversas técnicas de avaliação da audição, dentre elas o audiômetro pediátrico, marca Interacoustics, modelo PA2, semelhante ao modelo
PA5. Neste estudo observaram que o grupo de crianças nascidas pré-termo apresentou desvio da normalidade, sendo que as crianças sem seqüela neurológica recuperaram a curva da normalidade por volta de nove a 13 meses de idade. Houve a possibilidade de estabelecer níveis mínimos de resposta a tom puro com reforço visual luminoso a partir dos seis meses de idade. Ainda comentaram que esses se apresentaram de 10 a $20 \mathrm{~dB}$ acima do descrito na literatura, provavelmente pela presença de ruído ambiental, uma vez que os teste foram feitos em ambiente sem tratamento acústico. Sugeriram que se considere a variável gênero nos estudos da audição. Os autores destacaram que o comportamento auditivo tem um caráter biológico, relacionado ao processo de maturação do Sistema Nervoso Central ${ }^{(18)}$.

O desenvolvimento auditivo normal de uma criança é representado pela atenção ao som dos zero aos cinco meses, procura da fonte sonora dos três aos seismeses, localização lateral no plano da orelha a partir dos cinco meses, localização indireta para baixo a partir dos nove meses, localização direta para baixo e indireta para cima a partir dos 12 meses e localização direta para cima a partir dos 18 meses $^{(12,18-19)}$.

Para que as avaliações subjetivas da audição de lactentes pudessem se tornar mais objetivas, diversas técnicas são utilizadas, dentre elas a técnica de distração ${ }^{(11-27)}$ e o treino prévio $^{(22)}$.

Ao descrever o procedimento de distração, onde o papel do examinador responsável pela distração é manter a atenção do lactente na linha média e garantir o retorno do mesmo para esta posição após a resposta, "o brinquedo utilizado deve ser interessante, mas nem tanto de modo a distrair totalmente o lactente, por outro lado, se o examinador ou o brinquedo não forem suficientemente interessantes, a probabilidade de falso-positivos será alta"(22).

"Para a avaliação de lactentes e de crianças pequenas é fundamental o treinamento prévio de todas as tarefas, isso com o objetivo de ensiná-los a executarem as tarefas solicitadas e manter o controle do comportamento"(21). No caso da pesquisa dos níveis mínimos de resposta esse treinamento prévio é realizado com um estimulo auditivo supraliminar.

Em nossa trajetória cientifica se faz necessária a busca de procedimentos que permitam investigar as habilidades auditivas em lactentes. Na literatura encontramos inúmeros estudos que enfatizam a avaliação eletrofisiológica ${ }^{(23-28)}$. No entanto, na literatura nacional ainda são poucos os trabalhos utilizando métodos comportamentais para a avaliação de lactentes.

As dificuldades na elaboração e aplicação de provas para a avaliação das habilidades auditivas em crianças são muitas, principalmente em como se agrupar sujeitos, devido a grandes diferenças individuais e/ou inabilidades para atender algumas tarefas; além disso, algumas variáveis podem interferir no desempenho da criança, como suporte familiar, idade e escolarização ${ }^{(21)}$.

Sendo assim, é evidente a necessidade de novos estudos que possibilitem conhecer o desenvolvimento das habilidades auditivas em lactentes, auxiliando não só em procedimentos de triagem e diagnóstico, como também no acompanhamento do desenvolvimento das habilidades auditivas. 
O presente estudo tem como objetivos investigar, em campo livre, o comportamento auditivo de lactentes ouvintes de seis a 24 meses de idade cronológica, quanto aos aspectos de duração da avaliação, nível mínimo de resposta e a análise critica do método utilizado.

\section{MÉTODOS}

Este trabalho foi iniciado após a aprovação do Comitê de Ética da Faculdade de Odontologia de Bauru, Universidade de São Paulo, conforme processo no 61/2003. Os responsáveis pelos participantes consentiram na realização da pesquisa e a divulgação de seus resultados.

Para a investigação dos níveis mínimos de respostas foi utilizado o audiômetro pediátrico portátil modelo PA5, marca Interacoustics.

Foram selecionados 30 lactentes, de ambos os gêneros, matriculados em berçários públicos e/ou particulares, sem alterações auditivas. Os participantes encontravam-se dentro da faixa etária de seis a 24 meses de idade.

Para a seleção dos participantes foram utilizados os seguintes critérios de inclusão e exclusão.

Critérios de inclusão:

- Idade cronológica entre seis e 24 meses;

- Não apresentar queixa auditiva;

- Não apresentar indicador de risco para perda auditiva;

- Não apresentar perda auditiva;

- Não apresentar alterações no desenvolvimento neuropsicomotor.

Critérios de exclusão:

- Apresentar alterações observadas na inspeção visual do meato acústico externo, que impeça a realização da avaliação auditiva;

- Apresentar na entrevista especifica algum dado que o impeça a realização da avaliação auditiva.

Primeiramente foi aplicada uma entrevista específica com o(s) responsável(is) de todos os participantes, contendo dados de identificação, antecedentes familiares, indicadores de risco para deficiência auditiva, idade gestacional, evolução clínica de cada criança, entre outros.

Após a entrevista foi realizada a inspeção visual do meato acústico externo, utilizando-se o otoscópio da marca Heine ${ }^{\circledR}$, a fim de observar se existia algum impedimento para a realização do exame.

Em seguida foi iniciada a avaliação audiológica, por meio da audiometria de reforço visual com a utilização do audiômetro pediátrico PA5, marca Interacoustics.

\section{Audiometria de Reforço Visual (Audiômetro Pediátrico PA5)}

A pesquisa foi realizada utilizando tons puros modulados (warble), nas frequiências de $500 \mathrm{~Hz}, 1000 \mathrm{~Hz}, 2000 \mathrm{~Hz}$ e $4000 \mathrm{~Hz}$ nas intensidades de 20 dBNA a 80 dBNA. Neste estudo, o PA5 foi executado de forma não seqüencial, ou seja, durante a avaliação não foi testada uma determinada frequiência até obter seu limiar, mas sim de forma alternada entre as quatro frequiências até se chegar no nível mínimo de resposta para cada uma delas. Segundo a literatura pesquisada, mudar a frequiência em cada tentativa mostrou ser mais efetivo para manter a atenção da criança durante o teste ${ }^{(17)}$.

Os participantes foram avaliados em campo livre, dentro de cabina acusticamente tratada. Cada participante foi colocado no colo do acompanhante, com equipamento a uma distância de $50 \mathrm{~cm}$ de cada orelha. O primeiro examinador ficou atrás do lactente operando o audiômetro portátil PA5 e o segundo examinador ficou de frente para o participante realizando a técnica de distração. A sala de teste era desprovida de estímulos visuais os quais poderiam se tornar competitivos.

A intensidade mínima que ocorria a resposta em cada frequiência era registrada na ficha de registro.

Para esta pesquisa os seguintes parâmetros foram selecionados $^{(17)}$ :

- O tipo de resposta considerada foi de virar a cabeça em direção à fonte sonora e ao reforço visual (estímulo luminoso).

- O tempo de intervalo para a resposta foi de aproximadamente cinco segundos para apresentação do reforço, do contrário, o reforço não era apresentado.

- O tempo de apresentação do mesmo era de três segundos.

- O tipo de sinal selecionado foi o warble (tom puro modulado).

- O tempo do exame foi registrado em minutos.

Todos os responsáveis pelos participantes assinaram o Termo de Consentimento Livre e Esclarecido.

\section{RESULTADOS}

Participaram deste estudo 30 lactentes com idade entre seis e 24 meses de idade e de ambos os gêneros.

A amostra de 30 lactentes foi subdividida em três grupos etários, com intervalos de seis meses para cada faixa etária ${ }^{(17)}$ : O Grupo I, de seis meses a 11 meses 29 dias, apresenta-se com 11 participantes; o Grupo II, de 12 meses a 17 meses 29 dias, com nove participantes e o Grupo III, de 18 meses a 24 meses, com 10 participantes.

Com relação aos dados estatísticos descritivos pudemos observar que a média das idades foi de 15,2 meses. No Grupo I a média foi de nove meses, no Grupo II foi de 14,4 meses e no Grupo III foi de 20,6 meses.

Em relação ao gênero, este estudo investigou 13 participantes do gênero masculino e 17 do gênero feminino.

Em todos os participantes foi realizada a inspeção do meato acústico externo e todos estavam aptos a realizar a avaliação audiológica.

A média dos níveis mínimos de audição encontrados, com o uso do audiômetro portátil PA5, nos diferentes grupos etários, orelhas e freqüências encontram-se descritos nas Tabelas 1 a 4 .

A Tabela 1 representa os dados estatísticos descritivos de ambas as orelhas, nos diferentes grupos etários em $500 \mathrm{~Hz}$.

A Tabela 2 representa os dados estatísticos descritivos de ambas as orelhas, nos diferentes grupos etários em $1000 \mathrm{~Hz}$.

A Tabela 3 representa os dados estatísticos descritivos de ambas as orelhas, nos diferentes grupos etários em $2000 \mathrm{~Hz}$. 
Tabela 1. Média, mediana e desvio padrão da orelha direita e esquerda dos diferentes grupos etários em $500 \mathrm{~Hz}$

\begin{tabular}{lcccccc}
\hline & \multicolumn{2}{c}{ Grupo I } & \multicolumn{2}{c}{ Grupo II } & \multicolumn{2}{c}{ Grupo III } \\
& OD & OE & OD & OE & OD & OE \\
\hline Média & 29,0 & 29,0 & 28,9 & 28,9 & 28,0 & 28,0 \\
Mediana & 30,0 & 30,0 & 30,0 & 30,0 & 30,0 & 30,0 \\
Desvio Padrão & 3,1 & 3,2 & 3,3 & 3,3 & 4,2 & 4,2 \\
\hline
\end{tabular}

Tabela 2. Média, mediana e desvio padrão da orelha direita e esquerda dos diferentes grupos etários em $1000 \mathrm{~Hz}$

\begin{tabular}{lcccccc}
\hline & \multicolumn{2}{c}{ Grupo I } & \multicolumn{2}{c}{ Grupo II } & \multicolumn{2}{c}{ Grupo III } \\
& OD & OE & OD & OE & OD & OE \\
\hline Média & 29,1 & 29,1 & 31,1 & 28,9 & 28,0 & 28,0 \\
Mediana & 30,0 & 30,0 & 30,0 & 30,0 & 30,0 & 30,0 \\
Desvio Padrão & 3,0 & 3,0 & 6,0 & 3,3 & 4,2 & 4,2 \\
\hline
\end{tabular}

Tabela 3. Média, mediana e desvio padrão da orelha direita e esquerda dos diferentes grupos etários em $2000 \mathrm{~Hz}$

\begin{tabular}{lcccccc}
\hline & \multicolumn{2}{c}{ Grupo I } & \multicolumn{2}{c}{ Grupo II } & \multicolumn{2}{c}{ Grupo III } \\
& OD & OE & OD & OE & OD & OE \\
\hline Média & 30,9 & 31,8 & 28,9 & 28,9 & 31,0 & 28,0 \\
Mediana & 30,0 & 30,0 & 30,0 & 30,0 & 30,0 & 30,0 \\
Desvio Padrão & 7,0 & 7,5 & 3,3 & 3,3 & 5,7 & 4,2 \\
\hline
\end{tabular}

A Tabela 4 representa os dados estatísticos descritivos de ambas as orelhas, nos diferentes grupos etários em $4000 \mathrm{~Hz}$.

Tabela 4. Média, mediana e desvio padrão da orelha direita e esquerda dos diferentes grupos etários em $4000 \mathrm{~Hz}$

\begin{tabular}{lcccccc}
\hline & \multicolumn{2}{c}{ Grupo I } & \multicolumn{2}{c}{ Grupo II } & \multicolumn{2}{c}{ Grupo III } \\
& OD & OE & OD & OE & OD & OE \\
\hline Média & 29,1 & 30,0 & 28,9 & 28,9 & 27,8 & 27,8 \\
Mediana & 30,0 & 30,0 & 30,0 & 30,0 & 30,0 & 30,0 \\
Desvio Padrão & 3,0 & 4,5 & 3,3 & 3,3 & 4,4 & 4,4 \\
\hline
\end{tabular}

Por meio do teste estatístico de Mann-Whitney U, não houve diferença estatisticamente significante entre os limiares do gênero feminino e do masculino $(\mathrm{p}=0,687)$ e nem entre a orelha direita e a esquerda $(\mathrm{p}=0,293)$. No teste estatístico de Kruskal-Wallis, foi possível comparar os limiares dos diferentes grupos etários e concluir que não houve diferença estatisticamente significante entre eles ( $p \geq 0,05$ ).

O tempo do exame variou de quatro a 15 minutos, com média de 8,2 minutos no Grupo I; de três a oito minutos, com média de cinco minutos no Grupo II; e de quatro a nove minutos, com média de 5,8 minutos no Grupo III.

A Tabela 5 mostra os dados estatísticos descritivos relativos ao tempo de duração do exame.

Tabela 5. Média, mediana e desvio padrão com relação ao tempo de duração do exame nos diferentes grupos etários

\begin{tabular}{lccc}
\hline & Grupo I & Grupo II & Grupo III \\
\hline Média & 8,2 & 5,0 & 5,8 \\
Mediana & 6,5 & 5,0 & 5,0 \\
Desvio Padrão & 4,6 & 2,1 & 1,7 \\
\hline
\end{tabular}

\section{DISCUSSÃO}

A literatura já mencionada sugere que a variável gênero seja levada em consideração, uma vez que em seu estudo encontraram que, em todas as faixas etárias estudadas, as crianças do gênero feminino apresentaram predomínio de respostas a tons puros de menor intensidade em relação às do gênero masculino, o que pode sugerir a existência de diferenças entre o desenvolvimento das crianças conforme o gênero $^{(18)}$. Mas este fato não foi confirmado pelo presente estudo, uma vez que não foi observada diferença estatisticamente significante entre os níveis mínimos de resposta do gênero feminino e do masculino, talvez em função do número de participantes.

Os dados encontrados neste trabalho foram comparados com aqueles encontrados por outros autores brasileiros ${ }^{(18)}$, sendo esta a única literatura encontrada com uma população nacional que utilizou e recomendou a avaliação audiológica infantil com um audiômetro pediátrico, portátil e com reforço visual ${ }^{(18)}$.

Na literatura, o termo "nível mínimo de resposta" é sugerido para descrever a menor intensidade em que a resposta ocorre, uma vez que, numa avaliação audiológica pediátrica, a melhora das respostas podem acontecer em função da maturação do Sistema Nervoso Central ${ }^{(29)}$. Quando utilizando o audiômetro pediátrico portátil, a utilização deste termo é reforçada, já que este tem apenas sete níveis de intensidade, não permitindo encontrar o limiar auditivo, mas sim a menor intensidade, dentre as disponíveis, em que a resposta ocorreu ${ }^{(18)}$.

Os níveis mínimos de resposta encontrados em outros estudos variam conforme a idade da criança, quando mais nova a criança é, mais alto é seu nível mínimo de resposta ${ }^{(16)}$. Os limiares encontrados com o VRA em lactentes de seis a 12 meses de idade são semelhantes ao longo da faixa etária e mostram grande confiabilidade quando comparados com limiares obtidos na mesma criança com mais idade ${ }^{(22)}$. No presente estudo pode-se observar essa semelhança entre as médias e medianas dos níveis mínimos de audição nos três grupos etários estudados.

Os níveis mínimos de resposta encontrados neste estudo encontram-se entre 20 e $40 \mathrm{dBNA}$, o que condiz com os achados da literatura nacional e internacional ${ }^{(12,17-18,30)}$.

Nossos resultados corroboram os dados de estudos anteriores $^{(22)}$, uma vez que todos os grupos (I, II e III) apresentam médias e medianas muito semelhantes. O Grupo II foi o que se mostrou mais homogêneo entre as freqüências estudadas; isto pode ser devido a fatores que influenciam uma avaliação audiológica infantil, tais como: o nível de desenvolvimento e a maturação neuronal, a atenção da criança à tarefa e sua motivação para responder ${ }^{(22)}$.

Por apresentarem menor idade, os lactentes do Grupo I têm menor desenvolvimento e maturação neuronal, além de tempo de atenção reduzido ${ }^{(7)}$. Já os do Grupo III podem ter menor motivação para responder ao teste, uma vez que a técnica do reforço visual já não é tão interessante nessa faixa etária, fazendo com que o desinteresse pelo reforço aconteça mais rapidamente. Sendo assim, nossos achados vão ao encontro dos demais trabalhos ${ }^{(13,18,22)}$. 
Os aspectos que tornam os Grupos I e III destoantes, de alguma forma, do Grupo II não inviabilizam o exame para estas faixas etárias, apenas requerem maior experiência dos avaliadores. A literatura relata que a experiência dos avaliadores nos testes torna mais fácil a visualização das respostas dadas pelos lactentes ${ }^{(30)}$.

O condicionamento, proposto para que haja melhor desempenho dos lactentes na avaliação audiológica, tem como objetivo ensinar previamente as tarefas propostas no estudo, procedimento este que nos assegura obter informações fidedignas quanto às habilidades auditivas do lactente; o que em nosso estudo também pode ser observado em todos os grupos ${ }^{(21)}$.

\section{CONCLUSÕES}

- O método mostrou-se eficiente para investigar os limiares de audibilidade na faixa etária pesquisada, não somente como um método de triagem, mas também para auxiliar a investigação do nível mínimo de audição, em campo livre, durante o processo diagnóstico e o acompanhamento do desenvolvimento das habilidades auditivas. Além de ser um procedimento de baixo custo e não requerer equipamentos especiais;

- A determinação do nível mínimo de resposta possibilitou a comparação entre os lactentes e contribuiu também para a comparação deste procedimento com outros utilizados no processo de avaliação audiológica infantil;

- É um teste efetuado em um curto período de tempo, não sendo necessário despender muito tempo para sua aplicação.

\section{AGRADECIMENTOS}

Agradecemos à Fundação de Amparo à Pesquisa do Estado de São Paulo (FAPESP) por ter acreditado neste trabalho e dado seu apoio financeiro.

\begin{abstract}
Purpose: To investigate the auditory behavior of hearing infants with ages ranging from six to 24 months, on the aspects of evaluation length, minimum level of response and critical analysis of the method. Methods: Thirty infants of both genders were divided in three age groups: Group I - 11 infants aged 6 to 11 months 29 days; Group II - 9 infants aged 12 to 17 months 29 days; Group III - 10 infants aged 18 to 24 months. Frequencies of 500, 1000, 2000 and $4000 \mathrm{~Hz}$ were bilaterally, non-consecutively evaluated in free field. Results: The median of the minimum auditory level was $30 \mathrm{~dB}$ in all frequencies and groups. Groups I and III showed higher variability of minimum auditory levels, while Group II was more consistent. Group I needed more time to be conditioned, showing tiredness, excitement and short attention span, resulting in more interruptions during the test. Group III was less motivated to respond, since the technique of visual reinforcement is not so interesting at this age range, which makes the reinforcement even less exciting. These aspects do not make the procedure inadequate; however they require more experienced examiners. Conclusions: The method was efficient to the studied age range, not only as a screening procedure but also as a method to help the investigation of the minimum auditory level, in free field, during diagnostic process.
\end{abstract}

Keywords: Auditory perception/methods; Infant behavior; Diagnostic techniques, otological; Photic stimulation; Infant

\title{
REFERENCIAS
}

1. Yoshinaga-Itano C. Benefits of early intervention for children with hearing loss. Otolaryngol Clin North Am. 1999;32(6):1089-102.

2. Downs MP, Yoshinaga-Itano C. The efficacy of early identification and intervention for children with hearing impairment. Pediatr Clin North Am. 1999;46(1):79-87.

3. Oliveira TT, Chiari BM, Azevedo MF. Respostas auditivas de neonatos para sons calibrados. Pró-Fono. 1994;6(2):8-13.

4. Delaroche M, Thiebaut R, Dauman R. Behavioral audiometry: protocols for measuring hearing thresholds in babies aged 4-18 months. Int $\mathrm{J}$ Pediatr Otorhinolaryngol. 2004;68(10):1233-43.

5. Lancioni GE, Coninx F, Smeets PM. A classical conditioning procedure for the hearing assessment of multiply handicapped persons. J Speech Hear Disord. 1989;54(1):88-93.

6. Shaw P, Nikolopoulos T. The effect of initial stimulus type for visual reinforcement audiometry. Int J Audiol. 2004;43(4):193-7.

7. Schmida MJ, Peterson HJ, Tharpe AM. Visual reinforcement audiometry using digital video disc and conventional reinforcers. Am J Audiol. 2003;12(1):35-40.
8. Magnusson L, Börjesson E, Axelsson AC. Visual reinforcement audiometry. Comparison of loudspeaker arrangements. Scand Audiol. 1997;26(4):247-51.

9. Nielsen SE, Olsen SO. Validation of play-conditioned audiometry in a clinical setting. Scand Audiol. 1997;26(3):187-91.

10. Keller FS. Aprendizagem: teoria do reforço. São Paulo: Epu; 1973.

11. Skinner BF. Sobre o behaviorismo. São Paulo: Cultrix; 1974.

12. Northern JL, Downs MP. Testes audiológicos clínicos de criança. In: Northern JL, Downs MP. Audição em crianças. 3a ed. São Paulo: Manole; 1989. p. 143-96.

13. Delfino TPM. O condicionamento na audiometria lúdica [Tese]. São Paulo: Pontifícia Universidade Católica de São Paulo; 2003.

14. Suzuki T, Ogiba Y. Conditioned orientation reflex audiometry: a new technique for pure tone audiometry in young children under 3 years of age. Arch Otolaryngol. 1961;74(2):192-8.

15. Liden G, Kankkunen A. Visual reinforcement audiometry. Acta Otolaryngol. 1969;67(2):281-92. 
16. Thompson G, Weber BA. Responses of infants and young children to behavior observation audiometry (BOA). J Speech Hear Disord.1974;39(2):140-7.

17. Schubert SH. Avaliação audiológica em crianças de 6 a 24 meses de idade utilizando a audiometria de reforço visual informatizada [tese]. São Paulo: Pontifícia Universidade Católica de São Paulo; 2000.

18. Azevedo MF, Vieira RM, Vilanova LCP. Desenvolvimento auditivo de crianças normais e de alto risco. São Paulo: Plexus; 1995

19. Soares E, Ribeiro R, Azevedo MF. Estudo dos níveis mínimos de resposta para estímulo verbal, ruído branco e tom puro em crianças de 5 meses a 22 meses de idade. Pró-Fono. 1998;10(1):30-3.

20. Jayarajan V, Nandi R, Caldicott B. An innovation in insert visual reinforcement audiometry in children. J Laryngol Otol. 2005;119(2):132-3.

21. Lopes AC. Percepção da fala: uma proposta de avaliação em crianças [tese]. Bauru: Hospital de Reabilitação de Anomalias Craniofaciais da Universidade de São Paulo; 2000.

22. Gravel JS, Hood LJ. Avaliação audiológica infantil. In: Musiek FE, Rintelmann WF, editors. Perspectivas atuais em avaliação auditiva. Barueri: Manole; 2001. p. 301-09.

23. Harney CL. Infant hearing loss: the necessity for early identification. Bol Assoc Med PR. 2000;92(9-12):130-2.
24. Ramos EG, Zaeyen EJB, Simpson DM, Infantosi AFC. Detecção da resposta auditiva no EEG de crianças utilizando técnicas no domínio da freqüência. Rev Bras Eng Biomed = Braz J Biomech. 2000;16(3):127-37.

25. Raineri GG, Coube CZV, Costa Filho OA, Alvarenga KF. Emissões otoacústicas evocadas - produto de distorção em neonatos audiologicamente normais. Rev Bras Otorrinolaringol. 2001;67(5):6448.

26. Iñíguez C R, Cevo E T, Fernández L F, Godoy B C, Iñíguez S R. Detección precoz de pérdida auditiva en niños con factores de riesgo. Rev Otorrinolaringol Cir Cabeza Cuello. 2004;64(2):99-104.

27. Purdy SC, Agung KB, Hartley D, Patuzzi RB, O’Beirne GA. The postauricular muscle response: an objective electrophysiological method for evaluating hearing sensitivity. Int J Audiol. 2005;44(11):625-30.

28. Lieu JE, Champion G. Prediction of auditory brainstem reflex screening referrals in high-risk infants. Laryngoscope. 2006;116(2):261-7.

29. Matkin ND. Assessment of hearing sensitivity during the preschool years. In: Bess FH, editor. Childhood deafness: causation, assessment, and management. New York: Grune \& Stratton; c1977.

30. Gliddon ML, Martin AM, Green RA. A comparison of some clinical features of visual reinforcement audiometry and the distraction test. $\mathrm{Br}$ J Audiol. 1999;33(6):355-65. 